Revista de Matemática: Teoría y Aplicaciones 2003 10(1-2) : 168-172

CIMPA - UCR - CCSS ISSN: 1409-2433

\title{
PROBLEMAS MATEMÁTICOS SUGERIDOS POR LA GENÉTICA Y LA ECOLOGÍA DE POBLACIONES
}

\author{
Guillermo Gómez Alcaraz*
}

Recibido: 1 Nov 2001

\begin{abstract}
Resumen
La finalidad del presente trabajo consiste en esbozar un paralelo entre las ecuaciones de la Física Matemática y problemas generados en otra rama del conocimiento que bien podría llamarse de la Biología Matemática. El trabajo se divide en 2 partes; en la primera se discute un problema del comportamiento de una población diseminada en una cierta área, y en la segunda se intenta desarrollar métodos numéricos en las integrales continuales, solución de problemas de dicho tipo.
\end{abstract}

Palabras clave: Biomatemáticas, genética de poblaciones, métodos numéricos.

\begin{abstract}
The main goal of the present work is to show a paralellism between the equations in Physics and Mathematics, and some problems generated in another branch which could be called Mathematical Biology. The article is divided in two parts; in the first one we discuss a behavior problem of a population disseminated in a certain area, and in the second part we try to develop numerical methods in continuous integrals, solution of problems of that type.
\end{abstract}

Keywords: Biomathemaics, population genetics, numerical methods.

Mathematics Subject Classification: 92D25, 60H10, 60H15, 60H30.

\section{Algunos problemas de genética de poblaciones}

\subsection{El modelo más simple de genética de poblaciones relacionado con ecuaciones estocásticas}

Se discutirán algunos aspectos matemáticos del fenómeno de la deriva génica [1], [2], bajo la condición de que la población considerada migra a lo largo de una región continua.

\footnotetext{
* Departamento de Matemática, Facultad de Ciencias, Universidad Nacional Autónoma de México. EMail: gomal@servidor.unam.mx
} 
Se considera una población formada por $N$ individuos que se aparean al azar (panmixia). Se pondrá atención en algún rasgo controlado por un sólo gene, no relacionado con el sexo, y que posee 2 alelos ( $a$ y $A$ ), en competencia. Se supondrá que

$$
\left\{\begin{array}{l}
p_{n} \\
q_{n}=1-p_{n}
\end{array}\right.
$$

son las frecuencias relativas con las que aparecen los alelos $a$ y $A$, en la $n$-ésima generación.

Con el fin de simplificar el modelo se considera que las generaciones no se traslapan y el tamaño de la población es constante e igual a $N$ y puesto que no se consideran efectos de selección fenotípica puede considerarse a la población gametoploide (esto es, formada sólo de gametos).

Se sabe que bajo apareamiento puro al azar el contenido genotípico de la población no cambia, es decir $p_{n+1}=p_{n}$, sin embargo, por lo finito de la población, la formación de la siguiente generación, resultará ser aleatoria. Bajo estas condiciones, si se considera a $p_{n}$ como la probabilidad de que aparezca el alelo $a$ en el correspondiente experimento, entonces

$$
p_{n+1}=p_{n}+\sqrt{\frac{p_{n}\left(1-p_{n}\right)}{N}} g_{n}
$$

donde $\left\{g_{n}\right\}_{n \in \mathbb{N}}$ es la sucesión de variables aleatorias independientes y normalizadas $\left(E g_{n}=\right.$ 0 y $\left.E g_{n}^{2}=1\right)$. El caso de un número grande de generaciones se acostumbra tomar en calidad de unidad de tiempo al necesario para la aparición de $N$ generaciones.

Si se toma un proceso aleatorio $w(t)$ con incrementos independientes, con $\Delta t=\frac{1}{N} \mathrm{y}$

$$
\Delta w(t)=g_{n} \sqrt{\Delta t}
$$

para un $N$ suficientemente grande, entonces a manera de aproximación continua a la ecuación en diferencias (1) se puede obtener la ecuación diferencial estocástica de Ito:

$$
d \xi(t)=\sqrt{\xi(t)[1-\xi(t)]} d w(t) .
$$

Entonces las probabilidades de transición correspondientes al proceso estocástico $w$ satisfacen la ecuación de difusión

$$
\frac{\partial u}{\partial t}=\frac{y(1-y)}{2} \frac{\partial^{2} u}{\partial y^{2}}, \quad(0 \leq y \leq 1)
$$

cuya solución es conocida y hay trabajos como [3], donde se discute el problema de la tendencia de la población a ser una población homocigótica.

\subsection{Un modelo menos simple}

Se considera ahora una población desconcentrada y dispersa en algunos puntos, entre los cuales son permisibles las migraciones. Se discutirá la variante más simple, cuando dichos puntos se encuentran a lo largo de una recta y el intercambio de migrantes ocurre entre los puntos vecinos más cercanos en iguales proporciones de manera que el tamaño 
de la población en cada punto se conserva constante. Con la notación adecuada se llega a la ecuación en diferencias correspondiente y se puede transitar a tiempos continuos. Tomando como segmento elemental de tiempo a una generación, se obtiene otra ecuación estocástica:

$$
d \xi_{k}=\alpha\left(\xi_{k+1}-2 \xi_{k}+\xi_{k-1}\right) d t+\sqrt{\frac{\xi_{k}\left[1-\xi_{k}\right]}{N_{k}}} d w_{k}
$$

y su correspondiente ecuación para las probabilidades de transición

$$
\begin{aligned}
\frac{\partial u}{\partial t}= & \frac{1}{2} \sum_{k} \frac{y_{k}\left(1-y_{k}\right)}{N_{k}} \frac{\partial^{2} u}{\partial y_{k}^{2}}+\alpha \sum_{k}\left(y_{k+1}-2 y_{k}+y_{k-1}\right) \frac{\partial u}{\partial y_{k}} \\
& \left(0 \leq y_{k} \leq 1\right)
\end{aligned}
$$

Se considera el modelo continuo, respecto al tiempo, como a la variable de posición. Supóngase además que $l=$ "distancia entre puntos vecinos", $\nu_{k}=\frac{N_{k}}{l}=$ "densidad lineal de población", y $\beta=\alpha l^{2}$. El análogo continuo de la ecuación (5) será la nueva ecuación estocástica:

$$
\begin{aligned}
d \xi(t, x)= & \beta \frac{\partial^{2} \xi(t, x)}{\partial x^{2}} d t+\sqrt{\frac{\xi(t, x)[1-\xi(t, x)]}{\nu(x)}} d w(t, x), \\
& (0 \leq x \leq b, 0 \leq \xi \leq 1)
\end{aligned}
$$

con las condiciones a la frontera naturales, que significarían la ausencia de flujo de genes a través de la frontera:

$$
\frac{\partial \xi(t, 0)}{\partial x}=\frac{\partial \xi(t, b)}{\partial x}=0
$$

Aquí $w$ es una función aleatoria de 2 variables $w=w(t, x)$, la cual respecto de $t$ tiene incrementos independientes, mientras que respecto a $x$ tiene valores independientes. Con base en las peculiaridades de la normalización $(w(t, x)$ es una aproximación continua de la magnitud $\frac{w_{k}}{\sqrt{l}}$ ) esta función resulta no ser usual, sino generalizada (esto es, una distribución en el sentido de Schwartz). Todo esto muestra que $w(t, x)$ puede ser considerada como un proceso aleatorio de Wiener infinito dimensional, correspondiente a un espacio de Hilbert $H=L^{2}(0, b)$, pero concentrado en cierto espacio más amplio constituido por funciones generalizadas.

Sin embargo la solución $\xi$ de la ecuación estocástica (7) deberá ser una función usual de 2 variables $\xi=\xi(t, x)$, entre otras debido a su interpretación biológica. Si se toma

$$
\sqrt{\frac{\xi(t, x)[1-\xi(t, x)]}{\nu(x)}} d w(t, x) \stackrel{\text { not }}{=} f(t, x) d t
$$

de (7) se obtiene una ecuación de difusión no homogénea, con las condiciones a la frontera:

$$
\left\{\begin{array}{l}
\frac{\partial \xi}{\partial t}=\beta \frac{\partial^{2} \xi}{\partial x^{2}}+f \\
\frac{\partial \xi(t, 0)}{\partial x}=\frac{\partial \xi(t, b)}{\partial x}=0
\end{array}\right.
$$


cuya solución puede representarse como

$$
\xi(t, x)=\int_{0}^{b} \Gamma(t, x, z) \xi(0, z) d z+\int_{0}^{t} \int_{0}^{b} \Gamma(t-\tau, x, z) f(\tau, z) d z d \tau
$$

donde $\Gamma(t, x, z)$ es la función de Green del Problema (10), o con la notación original

$$
\begin{aligned}
\xi(t, x)= & \int_{0}^{b} \Gamma(t, x, z) \xi(0, z) d z+\int_{0}^{t} \int_{0}^{b} \Gamma(t-\tau, x, z) \times \\
& \times \sqrt{\frac{\xi(\tau, z)[1-\xi(\tau, z)]}{\nu(z)}} d_{\tau} w(\tau, z) d z
\end{aligned}
$$

donde a su vez $d_{\tau} w(\tau, z)$ requiere de cierta formalización. Esto lleva a la necesidad de ser más precisos y plantearse la ecuación estocástica (7) como:

$$
d \xi=A \xi d t+B(\xi) d w
$$

mientras que la ecuación integral (12) queda de la forma

$$
\xi(t)=e^{A t} \xi_{0}+\int_{0}^{t} e^{A(t-\tau)} B(\xi(\tau)) d w(\tau) .
$$

Se puede considerar a esta ecuación integral (12) prioritaria, con base en que posee sólo operadores acotados.

Desde el punto de vista estríctamente matemático se le puede dar la vuelta a tales dificultades, mientras que para la distribución de probabilidades se deduce la ecuación inversa de Kolmogorov, la cual representa una ecuación en derivadas variacionales:

$$
\frac{\partial u}{\partial t}=\frac{1}{2} \int_{0}^{b} \frac{\varphi_{\delta}(y(x))}{\nu(x)} \frac{\delta^{2} u}{\delta y(x)^{2}} d x+\beta \int_{0}^{b} y^{\prime \prime}(x) \frac{\delta u}{\delta y(x)} d x .
$$

Aquí el problema consiste en fundamentar el paso al límitecuando $\delta \rightarrow 0$, el cual llevaría a la ecuación de la distribución del proceso buscado $\xi(t, x)$ :

$$
\frac{\partial u}{\partial t}=\frac{1}{2} \int_{0}^{b} \frac{y(1-y)}{\nu(x)} \frac{\delta^{2} u}{\delta y(x)^{2}} d x+\beta \int_{0}^{b} y^{\prime \prime}(x) \frac{\delta u}{\delta y(x)} d x .
$$

Sin embargo en este paso se necesita una discusión complementaria del sentido biológico del suavizamiento descrito, que cambia el carácter del comportamiento de los procesos estudiados en los puntos $\xi=0$ y $\xi=1$, que juegan un papel particularmente importante al estudiar el problema de la tendencia a una población a la hómocigótica. 


\section{Aplicación de la integración funcional a modelos ecológi- $\cos$}

Al estudiar la dinámica de una población bajo distintas condiciones se presta para estudiar ecuaciones diferenciales no lineales suficientemente complicadas. Al agregarle efectos como el relacionado con la migración, estas ecuaciones como vimos en la primera parte se convierten en ecuaciones semejantes a ciertas ecuaciones de la Física Matemática. Estamos estudiando la manera de abordar tópicos relacionados con el tratamiento numérico de tales tipos de ecuaciones.

Esto lleva a la idea de aplicar en ciertos casos los mismos métodos numéricos semejantes a los que aplican los físicos. Se puede ilustrar tal aplicación a ecuaciones lineales, a no lineales y a sistemas de ecuaciones.

\section{Referencias}

[1] Feller W, (1950) An Introduction to Probability Theory and its Applications. Wiley, New York.

[2] Karlin, S.; Taylor, H.M. (1975) Basic Stochastic Processes Theory. Academic Press, New York

[3] Moran, P. (1973) Statisticheskie Protsessy Evulyutsionnoi Teorii. Moscú, Nauka.

[4] Ito, K. (1960) Veroyatnostnye Protsessy, Vyp. I, II, trad. del Japonés por Ventsel, A. y Dynkin, E., IL, Moscú.

[5] Strook, D.; Varadham, R.S. (1969) "Diffusion processes with continuous coefficients 1, 2", Communs. Pure and Appl. Math. 22: 345-400, 479-530.

[6] Trotter, H.F. (1959) "On the product of semi-groups of operators", Proc. Am. Math. Soc. 10(4): 545-551.

[7] Kac, M. (1951) "On some connections between probability and integral equations", Proc. 2nd Berkeley Sympos. Math. Stat. and Probab., Berkeley: 189-215. 